\title{
Telephone communication on the ICU: what nurses say to families
}

\author{
N Sangala, S Horscraft, A McGowan, F Baldwin* \\ From ESICM LIVES 2015 \\ Berlin, Germany. 3-7 October 2015
}

\begin{abstract}
Introduction
Families of patients consider timely, clear, and compassionate communication as an indicator of quality care in the ICU(1). Good communication with the family members of patients dying on the ICU can reduce the burden of bereavement, whilst better communication throughout the admission can improve the family experience of ICU in general (2). Nursing staff are major contributors to family communication through their frequent interactions at the bedside. Nursing staff also provide support to family members over the telephone.
\end{abstract}

\section{Objectives}

We performed a prospective observational study to understand the specific needs of family members during telephone calls with ICU nurses, and the ability of nurses to meet those needs.

\section{Methods}

We developed a questionnaire that was completed by nursing staff who had received telephone calls from the family and friends of patients over a one week period. The questionnaire was specifically designed to identify the needs of the caller, and additionally record the time and length of the call, the accessibility of the nurses, and record any adverse events that occurred during the telephone conversation.

\section{Results}

During the one-week period 66 questionnaires were returned of which 2 were incomplete and not used for analysis. Family members made the majority of calls with $31 \%$ coming from the patients' partner, $31 \%$ from their children, and $20 \%$ from other family members. Carers, police, alcohol liaison services and friends made the remaining calls. $21 / 64$ calls were made out of hours yet $87 \%$ of callers reached the nurse at their first try. Conversations lasted between 2-10 minutes at an average of 4.3 minutes. The only adverse event reported was the loss of a single NG tube. The clinical condition of patients was the primary focus in $38 \%$ of calls followed by emotional support in $13 \%$ of calls. In a further $36 \%$ of calls, both clinical condition and emotional support were the predominant focus of the conversation. Non-clinical information dominated only 5 of 64 conversations and nurses rarely took time to gather information regarding their patient from family members $(14 / 64)$. On 6 occasions the nursing staff were unable to answer all of the questions posed to them due to the requesting of detailed scan results (3/6), questions of prognostication (1/6), confidentiality issues (1/6), and a non-clinical explanation of visiting times $(1 / 6)$.

\section{Conclusions}

The predominant needs of family during telephone conversations with ICU nurses are the desire for clinical information and the need for emotional support. Nurses are readily accessible at all times of the day and provide a valuable source of information and support for family members over the telephone.

\section{Published: 1 October 2015}

\section{References}

1. In their own words: patients and families define high-quality palliative care in the intensive care unit: Crit Care Med 2010, 38(3):808-818.

2. A communication strategy and brochure for relatives of patients dying in the ICU: NEJM 2007, 5:469-478.

doi:10.1186/2197-425X-3-S1-A713

Cite this article as: Sangala et al.: Telephone communication on the ICU: what nurses say to families. Intensive Care Medicine Experimental 2015 3(Suppl 1):A713. 